\title{
THE EFFECT OF INJECTION OF THE RECOMBINANT HUMAN GROWTH HORMONE INTO INDUCED TEMPOROMANDIBULAR JOINT ARTHRITIS (AN EXPERIMENTAL STUDY)
}

\author{
Mohammed A. Altam ${ }^{1}$ BDS, Samraa A. El-Sheikh ${ }^{2} P h D$, Riham M. Eldibany ${ }^{2} h D$, Nesma M. \\ Khalil $^{3}$
}

\begin{abstract} and less systemic side effects. temporomandibular joint (TMJ). in control group a mean $\pm \mathrm{SD}=496.67 \pm 54.93 \mu \mathrm{m}$. regeneration of cartilage and bone.

1 B.D.S. Faculty of Dentistry, University of Mosul, Iraq.

2Professor of Oral and Maxillofacial Surgery, Faculty of Dentistry, Alexandria University, Egypt.

3Lecturer of Oral Biology Department, Faculty of Dentistry, Alexandria University, Egypt.
\end{abstract}

INTRODUCTION: Recombinant human growth hormone ( $\mathrm{rHGH}$ ) plays a central role for several metabolic functions including remodeling of bone and cartilage differentiation. Local administration of growth hormone (GH) could be more reasonable due to its more specific effects

OBJECTIVES: This study was designed to evaluate histological effects of intra-articular HGH injection in induced arthritis in rabbits

MATERIALS AND METHODS: This study was conducted on 12 healthy adult white New Zealand rabbits. TMJ arthritis was induced by injecting Complete Freund's Adjuvant (CFA) into TMJ bilaterally. The left side was considered as the study group and injected by rHGH into TMJ, while the right side was considered as the control group and sterile distilled water was injected into TMJ.

RESULTS: The condylar cartilage appeared relatively thick \& homogenous allover most of the condylar surface in the study group a mean \pm $\mathrm{SD}=825.92 \pm 61.09 \mu \mathrm{m}$ while,the condylar cartilage showed marked decrease in cartilage thickness \&disturbance in the osteochondral junction

CONCLUSIONS: From the current study, it can be concluded that the treatment of TMJ arthritis by rHGH leads to a better condition in

KEYWORDS: TMJ, arthritis, intra-articular injection, human growth hormone, Complete Freund's Adjuvant.

\section{INTRODUCTION}

Temporomandibular joint is a unique joint because it is a diarthroidal joint that allows a wide range of movements, but it is controlled by the masticatory muscles, ligaments and a specialized dense fibrous capsule. Any impairment of these structures is considered as temporomandibular disorder (1).

TMJ is reported to be involved in about $70 \%$ of all arthritis cases. Clinical signs and symptoms may include stiffness, ear and pre-auricular pain, joint clicking, crepitation, swelling, tenderness and mouth opening limitation (2).

Generally arthritis can be subdivided into three types: rheumatoid arthritis (RA), osteoarthritis (OA) and gouty arthritis. OA is a wear and tear type of arthritis with symptoms of pain and degeneration of bones. Whereas RA is a systemic chronic inflammatory disease that characteristically involves the synovial tissues (3).

The etiology of RA is multifactorial, with genetic and environmental components that together lead to early immune disturbance in both the innate and adaptive compartments and subsequent chronic inflammation (4).

Humans and other animals growth, cell reproduction and regeneration are stimulated by growth hormone (GH) which is a peptide hormone that is known as somatotropin or somatropin stimulates (5).

Human growth hormone (HGH) is considered as one of the most important factors that play an important role in craniofacial growth which depends on complex interactions between genes, nutrients, hormones and epigenetic factors (6).

GH plays a defined role in bone and cartilage metabolism, this can be mediated directly or indirectly through insulin like growth factor (IGF-I), although the mechanisms have not yet fully been Identified. GH is intermittently released from the anterior pituitary gland (7, 8).

It has been shown that bone formation in rats is promoted by the systemic administration of GH. GH receptors have been detected in various parts of rat's TMJ (9).

GH has no specific target organ. However, GH treatment stimulates mitotic activity and delays cell maturation in the mandibular condylar cartilage, so it has a profound effect on cartilage growth (10).

There are many non-surgical approaches that have been proposed through the years for treatment of TMJ rheumatoid arthritis such as occlusal splints and/or functional orthodontic appliances therapy, physiotherapy, pharmacotherapy, and arthrocentesis (11).

Drug local administration offers many advantages over systemic delivery, including increased bioavailability, reduced systemic adverse events, and lower total drug cost. Because cartilage is avascular, intra-articular therapy improves delivery to the cartilage. There are many intraarticular therapeutics such as; corticosteroids, hyaluronates, analgesics, platelet rich plasma (PRP) and cell based therapies (12).

Therefore, this study was conducted to evaluate the histological effects of intra-articular growth hormone injection (IAGHI) on the condylar bone and cartilage (repair) in induced TMJ arthritis in rabbits. 


\section{MATERIALS AND METHODS \\ Materials \\ Experimental animals}

This study was conducted on 12 healthy adult white New Zealand rabbits (age approximately 2-2.5 months and weighing 2-3 Kgs).

The experimental study was done in the animal house of the Faculty of Agriculture, Alexandria University.

TMJ arthritis was induced by injecting Complete Freund's Adjuvant (CFA) into TMJ bilaterally. Then they were left for 12 days.

Study group: $2 \mathrm{mg} / \mathrm{kg} / \mathrm{ml} \mathrm{rHGH}$ was injected into the left TMJ every other day for 12 days.

Control group: $1 \mathrm{ml}$ sterile distilled water was injected into the right TMJ every other day for 12 days.

All procedures were performed in accordance with a protocol approved by the Research Ethics Committee of Faculty of Dentistry, Alexandria University, Egypt.

\section{Drugs}

Complete Fraund's Adjuvant (CFA) is in the form of cell suspension $10 \mathrm{ml}$ ampule. It is composed of $5 \mathrm{mg}$ killed Mycobacterium butyricum in $1 \mathrm{ml}$ of paraffin oil (Complete Fruend's Adjuvant, Produced by Sigma Co., St. Louis, MO, USA. Imported by Egyptian International Center for Import) (13). Fig. (1A)

Recombinant human growth hormone (RHGH) is in the form of vial containing lyophilized powder $4 \mathrm{IU} /$ vial with diluent $2 \mathrm{ml}$ bacteriostatic water for injection containing benzyl alcohol as a preservative $0.9 \%$. RHGH vial was reconstituted prior to use with $2 \mathrm{ml}$ of accompanied diluent. Fig. (1B)
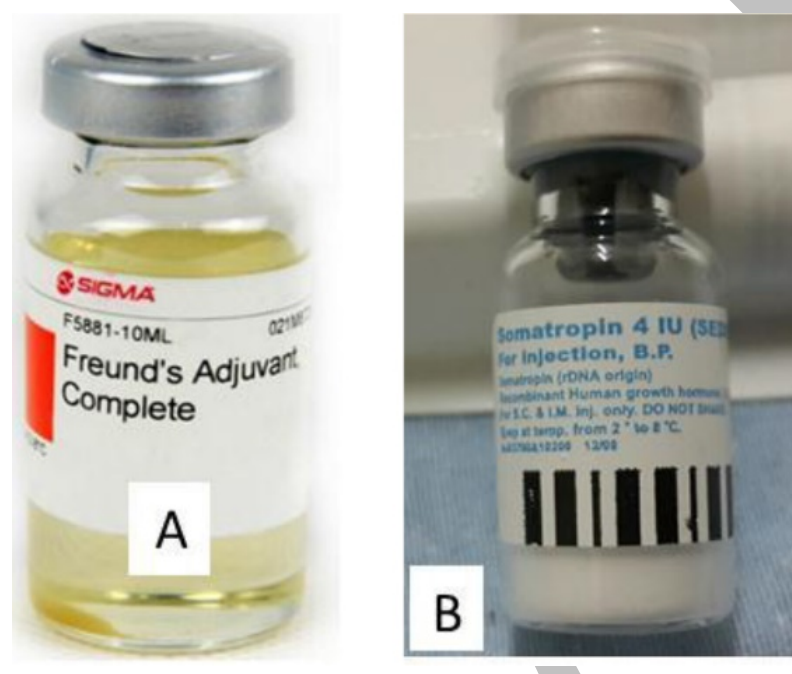

Figure 1: A. Complete Freund's Adjuvant.B. rHGH.

\section{Methods}

\section{Preoperative care}

The animals were examined by a veterinarian to exclude any diseased animal.

Animals were caged under standard laboratory and environmental conditions.They were weighed to estimate the amount of anesthetic and GH drugs. They were isolated for a week in a separate cage before being used in the experiment to acclimate them to housing and diet. They were allowed free access to tap water and were fed a commercial dry rabbit diet.

\section{Operative Phase \\ Anesthesia}

General anesthesia was induced by intramuscular injections of Ketamine $10 \mathrm{ml}$ vial (50mg/kg) (ketamar; $10 \mathrm{ml}$ vial produced by: Amoun Pharmaceutical Co. Egypt) and Xylazine $25 \mathrm{ml}$ vial (10mg/kg) (Xyla-ject; $25 \mathrm{ml}$ vial produced by: Adwia Pharmaceutical Co. Egypt).

\section{Injection Procedure}

All injections were done under aseptic conditions for all rabbits.

All injections were conducted under general anesthesia. Rabbits TMJ areas were shaved and wiped with Povidoneiodine (Betadine U.S.P. 10 \% W/V. Manufactured by: the Nile Co for pharmaceuticals and Chemical industries Cairo - A.R.E. Licensed by Mundipharma AG -Basel Switzerland).

The injection point was in a depression marked $1 \mathrm{~cm}$ distal to the lateral orbital canthus along the line passing between the lateral orbital canthus and tragus of the ear.

TMJ arthritis was induced by injecting $0.125 \mathrm{ml}$ of CFA, into the TMJ bilaterally of both the control and study groups. Then they were left for 12 days (14).

Afterwards, in the study group, $2 \mathrm{mg} / \mathrm{kg} / \mathrm{ml}$ of $\mathrm{rHGH}$ was injected into the left TMJs every other day for 12 days (7).

While in the control group, $1 \mathrm{ml}$ sterile distilled water was injected into the right TMJs every other day for further 12 days.

\section{Postoperative phase}

Care of injection site by application of Betadine.No postoperative medications were administered.Rabbits were allowed free access to tap water and were fed a commercial rabbit diet.

\section{Follow up phase}

\section{A-Clinical evaluation}

Post injection follow up was done daily to assess any complications that may arise including signs of infection and edema.

\section{B-Histological evaluation}

Preparation of histological sections: all rabbits were sacrificed on the 32nd day from the beginning of CFA injection. The animals were sacrificed with an overdose of sodium pentobarbital.

Bilateral TMJ specimens were dissected, and placed in $10 \%$ neutral buffered formalin.

After decalcification with $8 \%$ formic acid, the samples were embedded in paraffin wax which were prepared into $4-5 \mu$ sections of the TMJ.

Sections were stained with hematoxylin-eosin (H\&E) and alcian blue stains (15).

\section{C-Statistical analysis}

\section{Histomorphometrical analysis}

Histomorphometric analysis of the mean cartilage thickness (maturative and hypertrophic zones) in different groups was measured using Image J 1.46 program.Steps of measuring mean mandibular condylar cartilage thickness were as follows:

From each specimen three sagittal sections were obtained at different standardized depths. From each section one photograph was taken at the middle part of the condyle anteroposterior using the same magnification power.

The thickness of condylar cartilage was measured using the image J program by choosing measure from analyze tab and the measurement was recorded. 
The procedure was repeated at three different points and the mean was obtained.

The same procedure was repeated for each of the other two sections of the same specimen and the mean was calculated.

1. All of the previous steps were repeated for each of the twelve specimens in each of the study and control groups.

2. A line $1 \mathrm{~mm}$ long was drawn on the slide and a photograph was taken using the same magnification power. The length of this line was measured using image J. This was used as a reference to convert the obtained values from pixels to millimeters by choosing set scale from analyze. Then the values were converted to micrometers.

3. The values were expressed as means \pm standard deviation. Histomorphometrical cartilage thickness analysis:

Histomorphometric analysis of the mean cartilage thickness (maturative and hypertrophic zones) in different groups was measured.Student t-test was used for comparing between the two groups $\mathrm{P}$ value is Statistically significant at $\mathrm{p} \leq 0.05$

\section{RESULTS}

\section{Light microscopic results}

\section{Study group}

In H\&E Staining, this group showed articular cartilage layers with increase in the thickness in comparison with the control group

A generalized appearance of regeneration of the nearly normal structural features of the articular cartilage was the prevailing finding. Figs. (2).

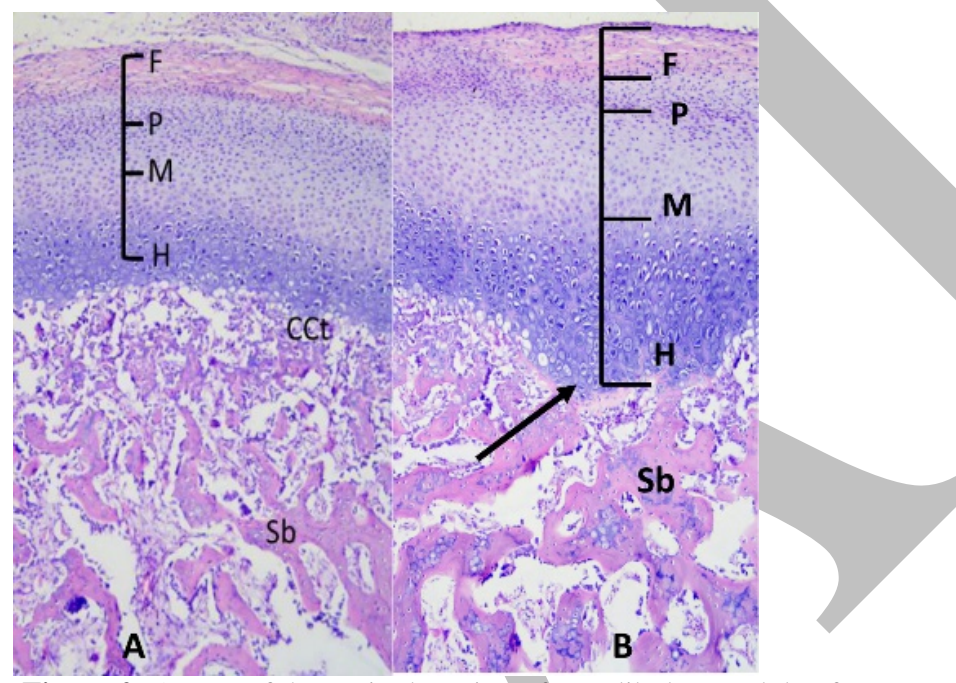

Figure 2: A. LM of the sagittal section of mandibular condyle of the study group showing the normal structural organization of the condyle. The condyle is covered with a fibrous articular layer (F). With an underlying prominent proliferative layer $(\mathrm{P})$. Zone of maturation (M) of chondrocytes is well recognized and the hypertrophic $(\mathrm{H})$ chondrocytes. CCt: calcified cartilage, (sb): Subchondral bone. (H\&E. x100). B. LM of the sagittal section of mandibular condyle showing a localized thickening of the hypertrophic chondrocytes $(\mathrm{H})$ \& slight invagination (arrows) osteochondral junction into the underlying Subchondral bone (sb). $\mathrm{F}$ : fibrous articular layer, $\mathrm{P}$ : proliferative layer, $\mathrm{M}$ : maturation zone \& H: hypertrophic zone. (H\&E. x100).

The condyle exhibited the nearly normal structural organization of all its layers including a fibrous articular layer (F), prominent zone of active cell proliferation (p), zone of maturing of chondrocytes (M) and finally the hypertrophied chondrocytes $(\mathrm{H})$. Few osteoclasts could be seen lining the endosteal surface of the subchondral bone.
Also strong alcian blue staining was noted Fig. (3) which reveals excesive glycose amino glycans of cartilage layers.

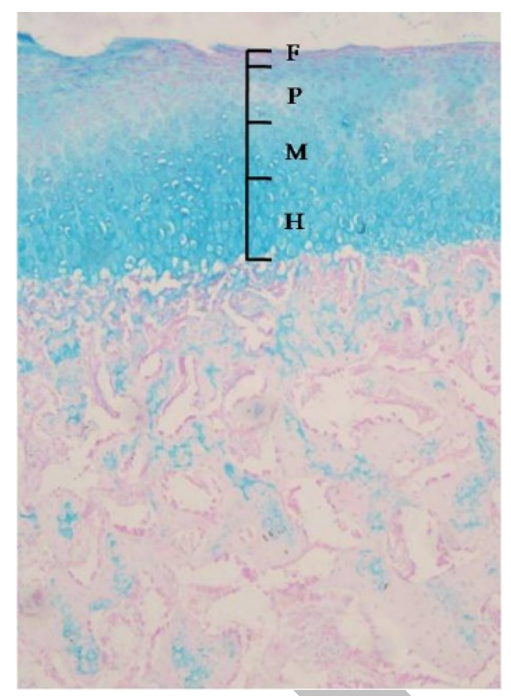

Figure 3: LM of the sagittal section of mandibular condyle in the study group showing relatively strong alcian blue-positive staining in the maturative $(\mathrm{M})$ and hypertrophic $(\mathrm{H})$ layers. F: fibrous articular layer, $\mathrm{P}$ : proliferative layer, $\mathrm{M}$ : maturation zone, $\mathrm{H}$ : hypertrophic zone (Alcian blue x100).

\section{Control group}

In H\&E Staining, the most obvious observation was the variation in condylar cartilage thickness all over the condylar surface. Some areas showed an irregular arrangement of chondrocytes. Disturbance in the osteochondral junction was evident by the presence of invagination of osteochondral junction into the subchondral bone. Figs. (4).

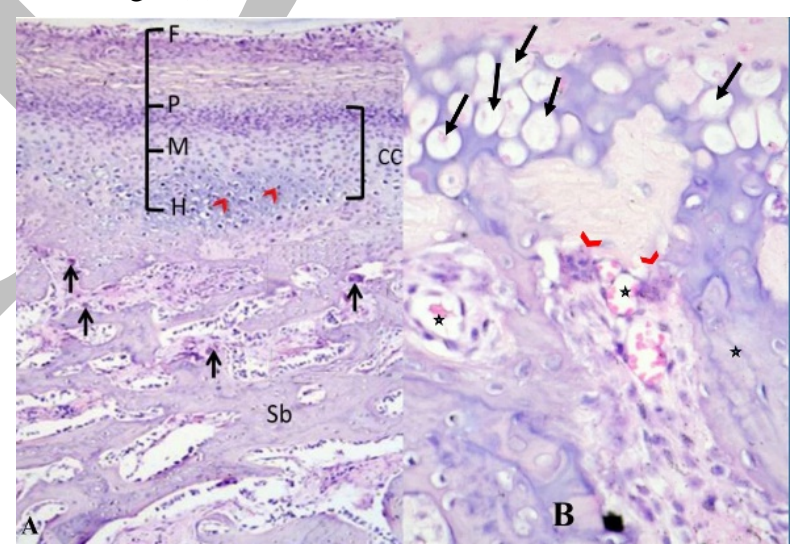

Figure 4: A. Light micrograph (LM) of the sagittal section of mandibular condyle in control group showing atrophy of some parts of the articular cartilage (thin arrow). Variation in the thickness of the condylar cartilage (cc) is evident. Some areas show invagination of osteochondral junction (thick arrow) into subchondral bone (sb). Note the vascularity (arrow heads) of the bone marrow. F: fibrous articular layer, P: proliferative layer, M: maturation zone, H: hypertrophic zone. (H\&E x100) B.LM of higher magnification of the sagittal section of mandibular condyle of the control group showing degenerated chondrocytes with empty lacunae (thick arrows), large multinucleated osteoclasts in Howship's lacunae (arrow heads) \& the blood capillaries (astisks) containing red blood cells (RBCs) and linead with flattened endothelial cells (H\&E. x100). 
Other areas showed advanced degree of condylar cartilage atrophy represented by degenerated chondrocytes with empty lacuanae. Many osteoclast cells could be seen on the outer surface of the bony trabeculae occupying Howship's lacunae. Also weak alcian blue staining was noted Fig. (5) which reveals dificient glycose amino glycans of cartilage layers
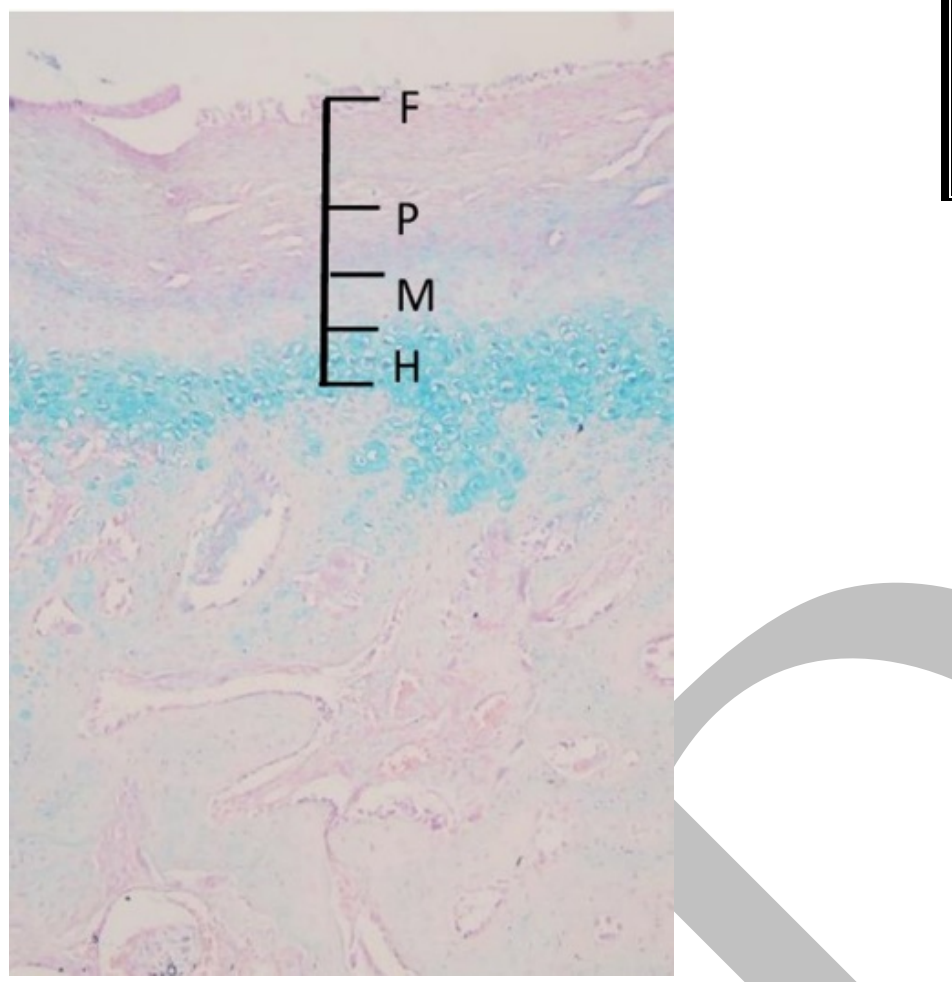

Figure 5: LM of the sagittal section of mandibular condyle of the control group showing weak Alcian blue-positive staining in the maturative (M) and hypertrophic (H) layers. F: fibrous articular layer, P: proliferative layer, $\mathrm{M}$ : maturation zone, $\mathrm{H}$ : hypertrophic zone (Alcian blue x100).

\section{Statistical analysis}

\section{Histomorphometrical cartilage thickness analysis:}

Histomorphometric analysis of the mean cartilage thickness (maturative and hypertrophic zones) in different groups was measured. Histomorphometric analysis of the mean cartilage thickness of study group showed marked increase in net cartilage thickness $(825.92 \pm 61.09 \mu \mathrm{m})$ compared to recorded mean of control group $(496.67 \pm 54.93 \mu \mathrm{m})$ and statistical significant $(\mathrm{P}<0.001$.). Fig. (6)

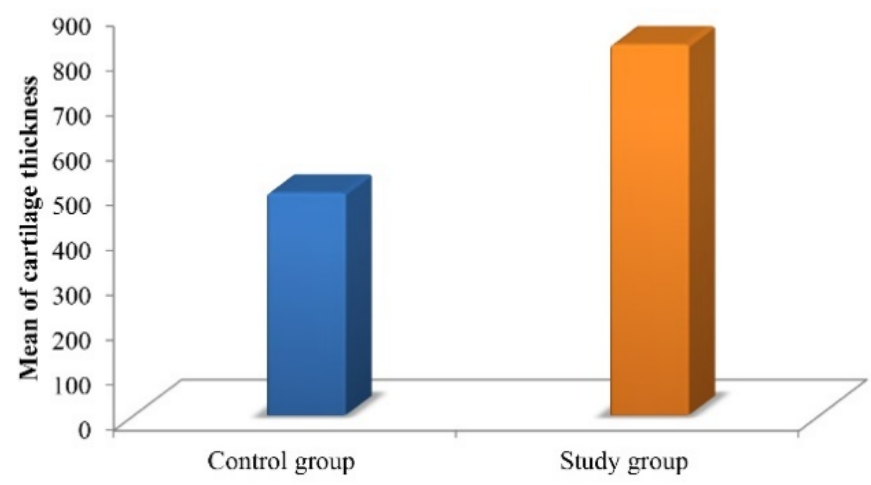

Figure 6: Comparison between the two groups according to the mean of cartilage thickness.
Table (1): Comparison between the two groups according to cartilage thickness.

\begin{tabular}{|c|c|c|c|c|}
\hline $\begin{array}{l}\text { Cartilage } \\
\text { thickness }(\mu \mathrm{m})\end{array}$ & $\begin{array}{l}\text { Control } \\
\text { group } \\
(n=12)\end{array}$ & $\begin{array}{l}\text { Study } \\
\text { Group } \\
(n=12)\end{array}$ & $\mathbf{t}$ & $\mathbf{P}$ \\
\hline Min.- Max. & $\begin{array}{l}401.0 \quad- \\
572.0\end{array}$ & $723.0-907.0$ & \multirow{3}{*}{13.884} & \multirow{3}{*}{$\begin{array}{l}<0.00 \\
1^{*}\end{array}$} \\
\hline Mean \pm SD & $\begin{array}{l}496.67 \\
\pm 54.93\end{array}$ & $\begin{array}{l}825.92 \pm 61.0 \\
9\end{array}$ & & \\
\hline Median & 500.0 & 843.50 & & \\
\hline
\end{tabular}

$\mathrm{t}, \mathrm{p}$ : $\mathrm{t}$ and $\mathrm{p}$ values for Student t-test for comparing between the two groups

*: Statistically significant at $\mathrm{p} \leq 0.05$

\section{DISCUSSION}

Arthritic TMJs are characterized by joint degeneration and facial pain that often lead to a significant amount of disability. They may be manifested, as pain, myalgia, headaches, and structural destruction known as degenerative joint disease (16). In early stages of TMJ arthritis there is articular chondrocyte cloning and subchondral bone erosion. In its later stages the synovial layers hypertrophy and extensive destruction of cartilage, disk and bone can be observed (17).

GH plays a defined role in bone and cartilage metabolism. However, GH treatment stimulates mitotic activity and delays cell maturation in the mandibular condylar cartilage, so it has a profound effect on cartilage growth $(7,9)$.

The rabbit has been widely used in models of TMJ arthritis and it is known to be a good model for human TMJ because of anatomical similarities and lateral physiological movements that do not exist in other mammals. Male rabbits were chosen in this study to prevent hormonal influence on cartilage and bone metabolism(18).

This study was conducted to evaluate the effects of intra-articular injection of rHGH on TMJ arthritis, induced by intra articular injection of CFA.

Holmdahl \& Kvick in 1992 (19) stated that the classical model of TMJ arthritis is induced by intradermal injection of CFA containing heat-killed mycobacteria and the arthritis develops within 2 weeks in susceptible rat strains. Intradermal injection into the parietal scalp induces arthritis in both TMJs and cervical vertebrae. Kuroki et al in 2011 (20) said that in the condyles of CFA-injection both bone formation and resorption occurred dynamically, so that the mechanism of arthritic destruction of the condyle is suitable to evaluate with this animal model.

In this study, the use of intra-articular injection of CFA instead of intradermal to get more destructive effect for all experimental animals.

The histological stain (H\&E) were used in this study in order to evaluate the effect of the recent route of treatment on structural components of the articular cartilage of TMJ as Zamma in 1983 (21) stated that arthritis in TMJ was more apparent histologically than clinically.

The histological results with $\mathrm{H} \& \mathrm{E}$ stain for the control group of this study were thinning and irregularity of some parts of the articular cartilage, severe atrophy in other parts of the cartilage, destruction of the subchondral bone and increase marrow space. 
Sousa et al in 2014 (22) agreed also with this study and noted a chronic systemic auto-immune inflammatory disease involving the breakdown of cartilage and adjacent bone tissue. Also they noted irregular and folded patterns on the joint surface that distinguished the limits between areas of normal and affected cartilage.

$\mathrm{Xu}$, et al in 2016 (23) investigated degenerative changes of TMJ during inflammation which occurs after injection of CFA in TMJ rats. The condyle became abnormally shaped and deviated from normal sagittal axis within the subchondral bone. Larger marrow cavities were observed in the 2 week after injection of CFA.

Lemos et al in 2016 (24) In their study, reported significant morphological changes in intra-articular injection of CFA in the left TMJ of the male Wistar rats, which include bone resorption of mandibular fossa, hyperplasia of the synovial membrane, infiltration of mononuclear inflammatory cells, and change in articular disc thickness.

In contradictory, George et al in 2013 (25) proved that fibrous articular lining of the head of the condyle thickened as a result of CFA injections . Thickening of the synovial lining has been reported with both adjuvant-induced and antigen induced TMJ arthritis. By adding simvastatin or steroid to the CFA injections, the thickness of the articular layer was reduced to the level of no treatment, supporting the anti-inflammatory properties of both drugs.

While in the study group of this study which treated with rHGH and stained with $\mathrm{H} \&$ E showed regenerated articular cartilage layers with increase in the thickness (Mean \pm SD = $421.20 \pm 40.04 \mu \mathrm{m})$ in comparison with the control group (Mean \pm SD $=825.92 \pm 61.09 \mu \mathrm{m}$ ) with statistical significant $\mathrm{p}$ value $<0.001$, normal architecture of the chondrocytes with signs of regenerative changes and the new subchondral bone trabeculae appeared.

Feizbakhsh et al in 2014 (7) confirmed this study results and said, it became apparent that HGH has a cleared stimulatory effect on the cartilage growth in rabbits treated with $2 \mathrm{mg} / \mathrm{kg} \mathrm{HGH}$ demonstrated significantly higher cartilage thickness .

Coincide with this study Hall in 2015 (26) mentioned that HGH promotes increasing size of the cells and increasing mitosis with development of increasing number of cells and specific differentiation of certain types of cells such as bone growth cells. Agreed with this study Lewinson et al in 1994 (27) proved that HGH was found to stimulate endochondral bone formation which would increase bone formation.

While the study group of present study showed almost increase in cartilage thickness with slight loss of metachromatism.

Kolbeck et al in 2003 (28) proved that administration of rHGH stimulates callus formation and ossification in the early stage of bone healing, which increased stiffness and mechanical strength.

\section{CONCLUSION}

From the current study, it can be concluded that treatment of TMJ arthritis by rHGH leads to a better condition in regeneration of cartilage and bone.

\section{CONFLICT OF INTEREST}

The authors declare that they have no conflicts of interest

\section{CONFLICT OF INTEREST}

The authors declare that they have no conflicts of interest.

\section{REFERENCES}

1. Piette E. Anatomy of the human temporomandibular joint. An updated comprehensive review. Acta Stomatol Belg. 1993;90:103-27.

2. Al-Mobireek A, Darwazeh A, Hassanin M. Experimental induction of rheumatoid arthritis in temporomandibular joint of the guinea pig: a clinical and radiographic study. Dentomaxillofac Radio. 2000;29:286-90.

3. Vashisst H, Gupta A, Jindal A, Jalhan S. Animal models for arthritis-a review. IJRAPR. 2012;2:20-5.

4. Asquith DL, Miller AM, McInnes IB, Liew FY. Animal models of rheumatoid arthritis. Eur J Immunol. 2009;39:2040-4.

5. Ranabir S, Reetu K. Stress and hormones. Indian J Endocrinol Metab. 2011;15:18.

6. Pirinen S. Endocrine regulation of craniofacial growth. Acta Odontologica Scandinavica. 1995;53:179-85.

7. Feizbakhsh M, Razavi M, Minaian M, Teimoori F, Dadgar S, Maghsoodi S. The effect of local injection of the human growth hormone on the mandibular condyle growth in rabbit. Dent Res J. (Isfahan) 2014;11:436-41.

8. Freedman RJ, Malkovska V, LeRoith D, Collins MT. Hodgkin lymphoma in temporal association with growth hormone replacement. Endocr J.2005;52:571-5.

9. Denko CW, Malemud CJ, editors. Role of the growth hormone/insulin-like growth factor-1 paracrine axis in rheumatic diseases. Semin Arthritis Rheum. 2005: Elsevier.

10. Maor G, Hochberg Z, Mark KVD, Heinegard D, Silbermann M. Human growth hormone enhances chondrogenesis and osteogenesis in a tissue culture system of chondroprogenitor cells. Endocrinology. 1989;125:1239-45.

11. Ramirez-Yanez GO, Young WG, Daley TJ, Waters MJ. Influence of growth hormone on the mandibular condylar cartilage of rats. Arch Oral Biol. 2004;49:58590.

12. Ringold S, Tzaribachev N, Cron RQ. Management of temporomandibular joint arthritis in adult rheumatology practices: a survey of adult rheumatologists. Pediatr Rheumatol Online J. 2012;10:26.

13. Vermeirsch H, Biermans R, Salmon PL, Meert TF. Evaluation of pain behavior and bone destruction in two arthritic models in guinea pig and rat. Pharmacol Biochem Behav. 2007;87:349-59.

14. Tominaga K, Alstergren P, Kurita H, Kopp S. Clinical course of an antigen induced arthritis model in the rabbit temporomandibular joint. J Oral Pathol Med. 1999;28(6):268-73.

15. Solaiman AAEM, Elagawany AM. Histological study of adult male albino rats' hepatocytes after formaldehyde administration and the possible protective role of dill oil. E J H. 2015;38:493-503.

16. Alhadlaq A, Mao J. Tissue-engineered neogenesis of human-shaped mandibular condyle from rat mesenchymal stem cells. J Dent Res. 2003;82:951-6.

17. Li P, Schwarz EM, editors. The TNF- $\alpha$ transgenic mouse model of inflammatory arthritis. Springer Semin Immunopathol. 2003: Springer. 
18. Ali AM, Sharawy MM. Histopathological changes in rabbit craniomandibular joint associated with experimentally induced anterior disk displacement (ADD). J Oral Pathol Med. 1994;23:364-74.

19. Holmdahl R, Kvick C. Vaccination and genetic experiments demonstrate that adjuvant-oil-induced arthritis and homologous type II collagen-induced arthritis in the same rat strain are different diseases. Clin Exp Immunol. 1992;88:96-100.

20.Kuroki Y, Honda K, Kijima N, Wada T, Arai Y, Matsumoto $\mathrm{N}$, et al. In vivo morphometric analysis of inflammatory condylar changes in rat temporomandibular joint. Oral Dis. 2011;17:499-507.

21.Zamma T. Adjuvant-induced arthritis in the temporomandibular joint of rats. Infect Immun. 1983;39:1291-9.

22. Sousa VR, Chagas Araújo Sousa F, Silva Filho OF, Rici G, Eli R, Neves Diniz A, et al. Comparative study by computed radiography, histology, and scanning electron microscopy of the articular cartilage of normal goats and in chronic infection with caprine arthritis-encephalitis virus. Microsc Res Tech. 2014;77:11-6.

23. Xu L, Guo H, Li C, Xu J, Fang W, Long X. A timedependent degeneration manner of condyle in rat CFAinduced inflamed TMJ. Am J Transl Res. 2016;8:556.

24. Lemos GA, Rissi R, de Souza Pires IL, de Oliveira LP, de Aro AA, Pimentel ER, et al. Low-level laser therapy stimulates tissue repair and reduces the extracellular matrix degradation in rats with induced arthritis in the temporomandibular joint. Lasers Med Sci. 2016;31:1051-9.

25. George MD, Owen CM, Reinhardt AL, Giannini PJ, Marx DB, Reinhardt RA. Effect of simvastatin injections on temporomandibular joint inflammation in growing rats. J Oral Maxillofac Surg. 2013;71:846-53.

26. Hall JE. Guyton and Hall Textbook of Medical Physiology E-Book: Elsevier Health Sciences; 2015.

27. Lewinson D, Bialik G, Hochberg Z. Differential effects of hypothyroidism on the cartilage and the osteogenic process in the mandibular condyle: recovery by growth hormone and thyroxine. Endocrinology. 1994;135(4):1504-10.

28. Kolbeck S, Bail H, Schmidmaier G, Alquiza M, Raun K, Kappelgard A, et al. Homologous growth hormone accelerates bone healing - a biomechanical and histological study. Bone. 2003;33:628-37. 